\title{
Trajectories of major depression in middle-aged and older adults: A population-based study
}

\author{
Xiaoling Xiang (D) I Jianjia Cheng
}

School of Social Work, University of Michigan, Ann Arbor, MI

\section{Correspondence}

Xiaoling Xiang, Assistant Professor, School of Social Work, University of Michigan, $1080 \mathrm{~S}$ University Ave, Ann Arbor, MI 48109.

Email: xiangxi@umich.edu

Funding information

National Institutes of Health, University of Michigan Older Americans Independence

Center Research Education Core, Grant/Award Number: AG024824
Objectives: This study aimed to examine depression trajectories and correlates in a nationally representative sample of middle-aged and older adults in the United States. Methods: The study sample consisted of 15661 participants aged over 50 years from the US Health and Retirement Study. Major depression was assessed using the Composite International Diagnostic Interview (CIDI-SF). Depression trajectories were identified using a group-based trajectory modeling enhanced to account for nonrandom attrition. Multinomial logistic regression was conducted to investigate predictors of depression trajectories.

Results: Four depression trajectory groups were identified: "never" (85.8\%), "increasing" (6.3\%), "decreasing" (3.2\%), and "persistently moderate/high" (4.7\%). Baseline depressive symptom severity was a strong predictor of depression trajectories. Older age, male sex, and non-Hispanic African American race were associated with a lower risk of the three trajectories with small to high depression burden, whereas chronic disease count was associated with a higher risk of these trajectories. The risk of being on the increasing trajectory increased with mobility difficulties. Difficulties in household activities predicted membership in the persistently moderate/high group.

Conclusions: A small but nonignorable proportion of middle-aged and older adults have chronic major depression. Initial symptom severity and chronic disease burden are consistent risk factors for unfavorable depression trajectories and potential targets for screening and intervention.

\section{KEYWORDS}

group-based trajectory modeling, Health and Retirement Study, major depression

\section{1 | INTRODUCTION}

Major depression occurs in $2 \%$ of adults 55 years or older ${ }^{1}$ and increases substantially among older adults in certain health and longterm care settings. ${ }^{2}$ Despite being less common in late life, major depression has a more chronic course and poorer prognosis in older adults than in younger adults ${ }^{3}$ and causes significant disability and impaired well-being. ${ }^{4}$ In addition, because of age-related increase in the risk of cerebrovascular disease, a significant proportion of late-life depression may fall under the category of vascular depression, which predicts a poorer course of depression. ${ }^{5}$
Evidence from population-based studies suggests that the longterm naturalistic trajectories of depressive symptoms are heterogenous across the life span, with most people experiencing few or no symptoms and a notable minority experiencing persistent symptoms. ${ }^{6}$ Studies focused on older adults have generated converging results and have typically identified 3-5 distinctive trajectory groups. ${ }^{7-15}$ Trajectory groups with greater symptom burden were associated with female sex, low income and education, limited social support, ${ }^{8,10,11,16,17}$ functional impairment, ${ }^{8,9,16}$ physical illnesses, ${ }^{8,9,12,14-16}$ and initial symptom severity. ${ }^{7,14,18}$ Difference in the shape of depression trajectories by age groups has also been 
reported, ${ }^{12,14,15}$ while findings regarding the impact of cognition have been mixed. ${ }^{8,9,19}$

Previous studies have focused on examining the course of depressive symptoms and have rarely investigated the naturalistic long-term trajectories of major depressive disorder in older adults, the prognosis of which is generally worse than that of minor depression. ${ }^{20}$ Most long-term follow-up studies of depressive disorders have involved mixed-age samples from specialty psychiatric inpatient or outpatient settings. Many older adults with depression do not seek treatment, and those who do may represent a more serious form of the disorder. For this reason, a population-based sample is considered the best data source for the study of natural development of depressive disorders. ${ }^{21}$ One of the first population-based studies of the long-term natural course of major depression, the Baltimore Epidemiologic Catchment Area Follow-up, found the course of major depression to be relatively stable from first to later episodes, and the researchers found few strong predictors of recovery or recurrence in a mixed-age sample during up to 15 years of follow-ups. ${ }^{21}$ Another study involving a mixed-age sample from the Netherlands identified five distinctive trajectories over 2 years among outpatients with major depression, dysthymia, or both, ranging from mild severity and rapid remission to high severity and chronic course. ${ }^{22}$ Older age predicted the poorest course in the Dutch study. ${ }^{22}$ Given the documented age differences in the etiology, diagnostics, and prognosis of depression, ${ }^{23}$ it is unclear whether the trajectories observed in younger adults would apply to older adults. The few studies that focused on older adults examined trajectories of depressive symptoms during follow-ups among those with major depression at baseline and did not report on the diagnostic status of major depression during follow-ups. ${ }^{24,25}$

The purpose of the present study was to identify trajectories of major depression in a population-based sample of middle-aged and older adults and to identify factors associated with the trajectories. The large and representative sample and extended follow-up periods provided a rare opportunity to observe the long-term naturalistic trajectories of major depression in late life. On the basis of the literature, we hypothesized a priori that there would be a large group of participants who never experienced major depression during the study period, ${ }^{6}$ and the rest would follow three trajectories characterized with a recovery, recurrent, and chronic course, respectively. ${ }^{26}$

\section{2 | METHODS}

\section{1 | Data}

The Health and Retirement Study (HRS) ${ }^{27}$ is a nationally representative study of people aged 51 years and older in the United States, sponsored by the National Institute on Aging (grant number NIA U01AG009740) and conducted by the University of Michigan. The HRS participants include several birth cohorts with varying entry times. For example, the initial 1992 HRS cohort, born in 1931 to 1941, was first interviewed in 1992, and the Children of the Depression (CODA) cohort, born in 1924 to 1930, was first interviewed in

\section{Key points}

- This was the first population-based study of long-term trajectories of major depression in a nationally representative sample of middle-aged and older adults in the United States.

- Changes in occurrences of 12-month major depression followed four trajectories: never, increasing, decreasing, and persistently moderate/high.

- Baseline depressive symptom severity was a strong and consistent predictor of depression trajectory groups.

1998. The HRS now employs a steady-state design, replenishing the sample every 6 years with younger cohorts. Biennial interviews with eligible individuals are conducted after the first baseline interview. More information is available at the HRS website: http://hrsonline. isr.umich.edu/.

The present study used data from 2008 through 2016 surveys (a total of five waves) and included HRS participants aged 51 years or older during the 2008 interview. Prior waves were not used because the HRS did not continuously administer the key depression measure of interest before 2008. A total of 17217 participants responded to the 2008 interview, including 1140 proxy respondents who did not receive the depression assessment. Excluding proxies ( $n=1140)$, persons with missing data on the key depression measure $(n=4)$, and persons younger than 51 years of age $(n=412)$, the final study sample consisted of 15661 middle-aged and older adults, with up to five interviews per person. Not all participants had five rounds of interviews due to attrition.

This study was a secondary data analysis of de-identified public data involving adults and was deemed as exempt human subjects research by the University of Michigan Institutional Review Board.

\section{$2.2 \mid$ Measures}

\subsection{1 | Major depression}

The World Health Organization's Composite International Diagnostic Interview (CIDI) is a fully standardized structured diagnostic interview designed for assessment of psychiatric disorders through large surveys with trained lay interviewers. The full CIDI has been validated against a trained clinical interviewer. ${ }^{28}$ The HRS included a short-form version of CIDI (CIDI-SF) for major depressive episode (MDE) that approximates the diagnosis given by the full CIDI. ${ }^{29}$ Unlike the full CIDI, which asks about lifetime experience, the CIDI-SF probes intensity and duration of symptoms of dysphoria and anhedonia in the past 12 months. CIDI-SF had a specificity of $93.9 \%$ and a sensitivity of $89.6 \%$ compared with the full CIDI. ${ }^{28}$ A score of greater than or equal to 5 on the CIDI-SF indicates five or more depressive symptoms in the same 2-week period over the last 12 months, which corresponds to 
the third edition of the Diagnostic and Statistical Manual of Mental Disorders (DSM-III-R) criteria for major depression.

\subsection{2 | Predictors of depression trajectories}

Predictors of depression trajectories were selected based on the earlier discussion of the risk factors identified from previous studies. Predictors were measured at the 2008 survey to minimize the possibility of reverse causality. Sociodemographic factors included age, sex, race/ethnicity, education, marital status, and household assets (sum of all wealth components minus all debt). The physical health indicator included total number of physician-diagnosed health conditions (hypertension, diabetes, heart disease, stroke, lung disease, cancer, and arthritis). Physical functioning indictors included difficulties in self-care (including bathing, dressing, and eating), household activities (instrumental activities of daily living [IADLs], including shopping for groceries, preparing hot meals, using the phone, managing money, and taking medications), and mobility (including walking one block, walking several blocks, walking across a room, climbing one flight of stairs, and climbing several flights of stairs). Having a difficulty was indicated by answering, "yes" or "cannot do" to the question "Do you have any difficulty with ..." Cognitive functioning was assessed using the Telephone Interview for Cognitive Status, including immediate and delayed word recall (memory test: 0-20 points), the Serial $7 \mathrm{~s}$ subtraction test (working memory test: 0-5 points), and backwards counting (mental processing speed test: $0-2$ points). ${ }^{30}$ A summary score of cognitive functioning ranged from 0 to 27 , with a higher score indicating better cognitive functioning. A score of 11 or below defines cognitive impairment based on the Weir-Langa Classifications. ${ }^{31}$ Baseline depressive symptom severity was measured using the eight-item Center for Epidemiologic Studies Depression Scale (CES-D). ${ }^{32}$ The eight-item CES-D score ranged from 0 to 8, with a higher score indicating more severe symptoms.

\section{3 | Analysis}

Trajectories of major depression were identified using a group-based trajectory modeling, enhanced to account for nonrandom attrition. ${ }^{33}$ The basic group-based trajectory modeling is a specialized application of finite mixture modeling aimed to identify clusters of individuals who follow similar progressions of some outcome over time. ${ }^{34}$ The basic group-based trajectory model assumes missing at random such that the probabilities of group membership assignment and attrition are independent, which can lead to biased estimates of trajectory group size when this assumption is violated. ${ }^{33}$ In the enhanced model, attrition is modeled simultaneously with the trajectory group as a function of time before dropping out using a logit distribution. Estimated probabilities of dropping out are specific to the trajectory groups.

A series of models were fitted by using the Proc Traj plug-in. ${ }^{35}$ The best fit model was selected based on the Bayesian information criteria (BIC), group distinctiveness and interpretability, and the average posterior probability of group assignment ${ }^{34}$ greater than 0.7. Participants were assigned to the trajectory group for which they had the highest posterior probabilities of group membership.

After determining group membership, we conducted descriptive statistics stratified by trajectory groups to describe and compare sample characteristics from the 2008 survey. Multinomial logistic regression was applied to predict depression trajectory group membership as a function of the 2008 sample characteristics. The 2008 HRS survey design factors (weights, strata, and clusters) were used in descriptive and regression analyses to generate nationally representative estimates using Taylor linearization for variance estimation. Analyses were conducted using Stata 15.1 (Stata Corp, College Station, TX).

\section{3 | RESULTS}

\section{1 | Depression trajectory groups}

A logit model with four trajectory groups was the best fit to the data based on changes in BIC values (see Table 1). Average posterior probability of group assignment was 0.78 (range: $0.73-0.81$ ) for group 1 , 0.71 (range: $0.45-0.94$ ) for group 2, 0.87 (range: 0.53-0.95) for group 3 , and 0.91 (range: $0.55-1.0$ ) for group 4. As shown in Figure 1, group 1 ("never") did not experience any 12-month MDE during the study period, best presenting $85.8 \%$ of the weighted sample. Group 2 ("increasing") experienced a slight but noticeable increase in the risk

TABLE 1 Tabulated Bayesian information criterion (BIC) and $2 \Delta B I C$

\begin{tabular}{|lllr|}
\hline Number of Groups & \multicolumn{1}{l}{ BIC } & Null model & 2( $\Delta \mathrm{BIC})$ \\
\hline 1 & -23408.24 & & \\
\hline 2 & -21841.36 & 1 & 3133.76 \\
\hline 3 & -21814.44 & 2 & 53.84 \\
\hline 4 & -21800.16 & 3 & 28.56 \\
\hline 5 & -21827.59 & 4 & -27.43 \\
\hline 6 & -21855.15 & 5 & -55.12 \\
\hline
\end{tabular}

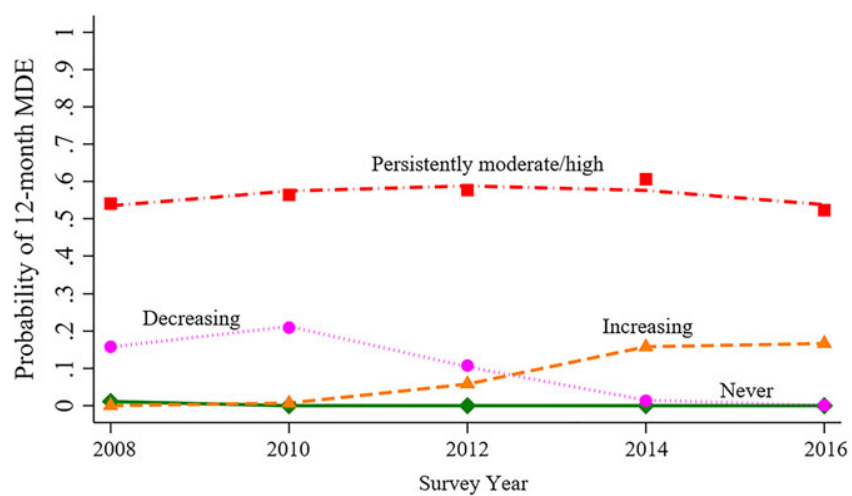

FIGURE 1 Depression trajectories over 8 years jointly modeled with attrition. This figure shows the estimated probability of experiencing a 12-month major depressive episode at each survey round for each trajectory group. $\mathrm{MDE}=$ major depressive episode [Colour figure can be viewed at wileyonlinelibrary.com] 
of 12-month MDE over time, best representing $6.3 \%$ of the weighted sample. Group 3 ("decreasing") captured people whose risk declined and became free of 12-month MDE at the end of the study period, best representing $3.2 \%$ of the weighted sample. Group 4 ("persistently moderate/high") represented a group of people who maintained moderate to high risk of 12-month MDE throughout the study period, best representing $4.7 \%$ of the weighted sample.

\section{2 | Sample characteristics by depression trajectories}

Depression trajectory groups differed significantly in sociodemographic backgrounds, physical health, physical functioning, and cognitive functioning. The "never" group had the highest share of men, non-Hispanic whites, and persons with a college degree; had the highest average age and total household assets; and had the lowest chronic disease and functional limitation counts. In contrast, the "persistently moderate/high" group had the lowest share of nonHispanic whites and persons with a college degree; had the lowest average age and total household assets; and had the highest chronic disease and functional limitation counts and proportion of persons with cognitive impairment (Table 2).

\section{3 | Factors shaping depression trajectories}

In multinomial logistic regression, baseline CES-D score and chronic disease count were associated with a higher relative risk of being on the three depression trajectories ("increasing," "decreasing," and "persistently moderate/high") versus the "never" trajectory. The relative risk of being on the "increasing," "decreasing," and "persistently moderate/high" trajectory decreased with age and was also lower among men than women and among non-Hispanic African Americans than whites. Mobility difficulties were associated with a higher relative risk of being on the "increasing" trajectory whereas household activities difficulties were associated with a higher relative risk of being on the "persistently moderate/high" trajectory. Cognitive impairment was associated with a lower relative risk of being on the "increasing" and "decreasing" trajectories (Table 3).

\section{4 | DISCUSSION}

The present study is one of the first population-based studies of the long-term trajectories of major depression in a nationally representative sample of middle-aged and older adults in the United States. Most middle-aged and older adults did not have major depression during the 8 years of follow-ups, while nearly $15 \%$ had elevated risk for a 12-month MDE during the study period. In line with previous studies, ${ }^{21,36,37}$ few predictors significantly distinguished between trajectory groups. Baseline depressive symptom severity was a strong predictor of trajectories with small to high burden of depression, in line with previous studies linking severity of index episode to persistent depression. ${ }^{38,39}$ The risk of increasing and persistently moderate/high depression trajectories tended to be lower for younger persons, men, non-Hispanic African Americans, and persons with higher socioeconomic status and was higher for persons with chronic diseases and functional limitations.

Using HRS data, Liang et al ${ }^{12}$ identified six trajectories of depressive symptoms assessed using the eight-item CES-D, with the majority of participants suffering very few symptoms. Findings from our study are similar in that the majority of participants did not experience 12month MDE during the study period. However, in Liang et al, African Americans were significantly more likely to be in trajectories of more elevated depressive symptoms, whereas in our study, African Americans were significantly less likely to be in trajectories of more elevated risk for major depression. Difference in model specification, particularly the adjustment of confounders, may explain this inconsistency. For example, an analysis of 1996 HRS data found that although African Americans exhibited elevated rates of major depression relative to whites in bivariate analysis, this trend was reversed after adjusting for potential confounders such that African Americans had a lower risk of major depression than whites. ${ }^{40}$ Another possible explanation is that racial difference in depression rates is dependent on the type of depression. A population-based study found that while prevalence of major depressive disorder was significantly higher in whites than in African Americans, the opposite pattern was found for dysthymic disorder. ${ }^{41}$

Consistent with reports from previous studies, ${ }^{36,42}$ our findings suggest that the natural course of major depression in late life is chronic and unremitting. In a sample of older Dutch adults where $71 \%$ met diagnostic criteria for major depression at baseline, over half of the study sample still had major depression at 2-year follow-up. ${ }^{36} \mathrm{~A}$ study of older psychiatric outpatients with depression diagnosis in Brooklyn, New York, reported even more dire outcomes: On followup interviews that ranged from 13 to 52 months, $85 \%$ of persons with subclinical depression at baseline, $90 \%$ of persons with clinical depression at baseline, and $62 \%$ of persons in remission at baseline were depressed. $^{42}$

The significant predictors of depression trajectories identified in our study are largely in line with those from previously cited studies. Our study adds to the literature by showing that different domains of functional limitations play different roles in shaping the trajectories of major depression. Difficulty in performing household activities was a strong predictor of having chronic moderate to high risk for MDE whereas mobility limitation was associated with an increase in the risk of MDE over time. Mobility limitations concerning a person's interaction with their environment and capacity to control the surrounding world, or "environmental mastery," are one of the six main components of psychological well-being. ${ }^{43}$ Having a mobility limitation, particularly in the extreme cases of completely homebound individuals, diminishes a person's environmental mastery and self-determination, adversely affecting their psychological well-being. Mobility limitation has been frequently linked to higher prevalence and incidence of depression in older adults. ${ }^{44,45}$ Items included in our mobility difficulty index (eg, walking across a room) are typically combined with other self-care activities (eg, dressing) to form an indicator of activity of daily 


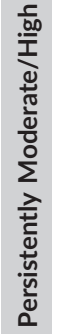

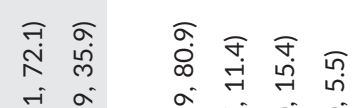

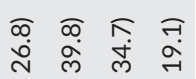

帒

苛突

ते

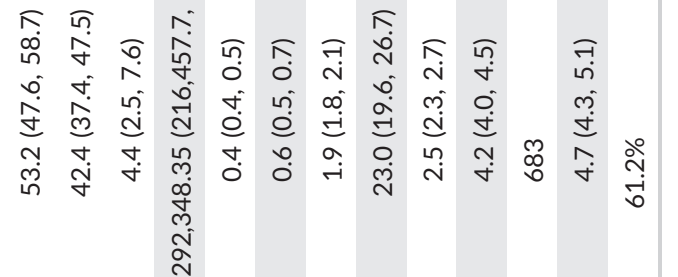

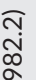

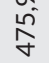

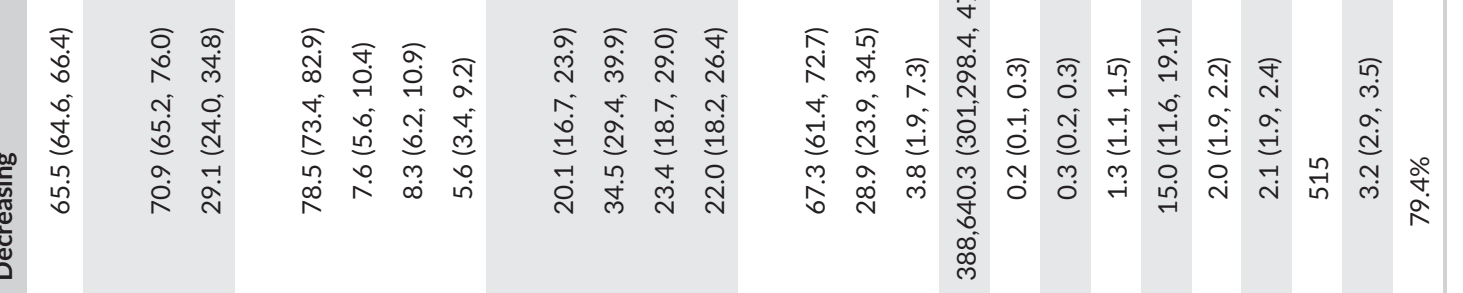

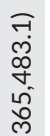

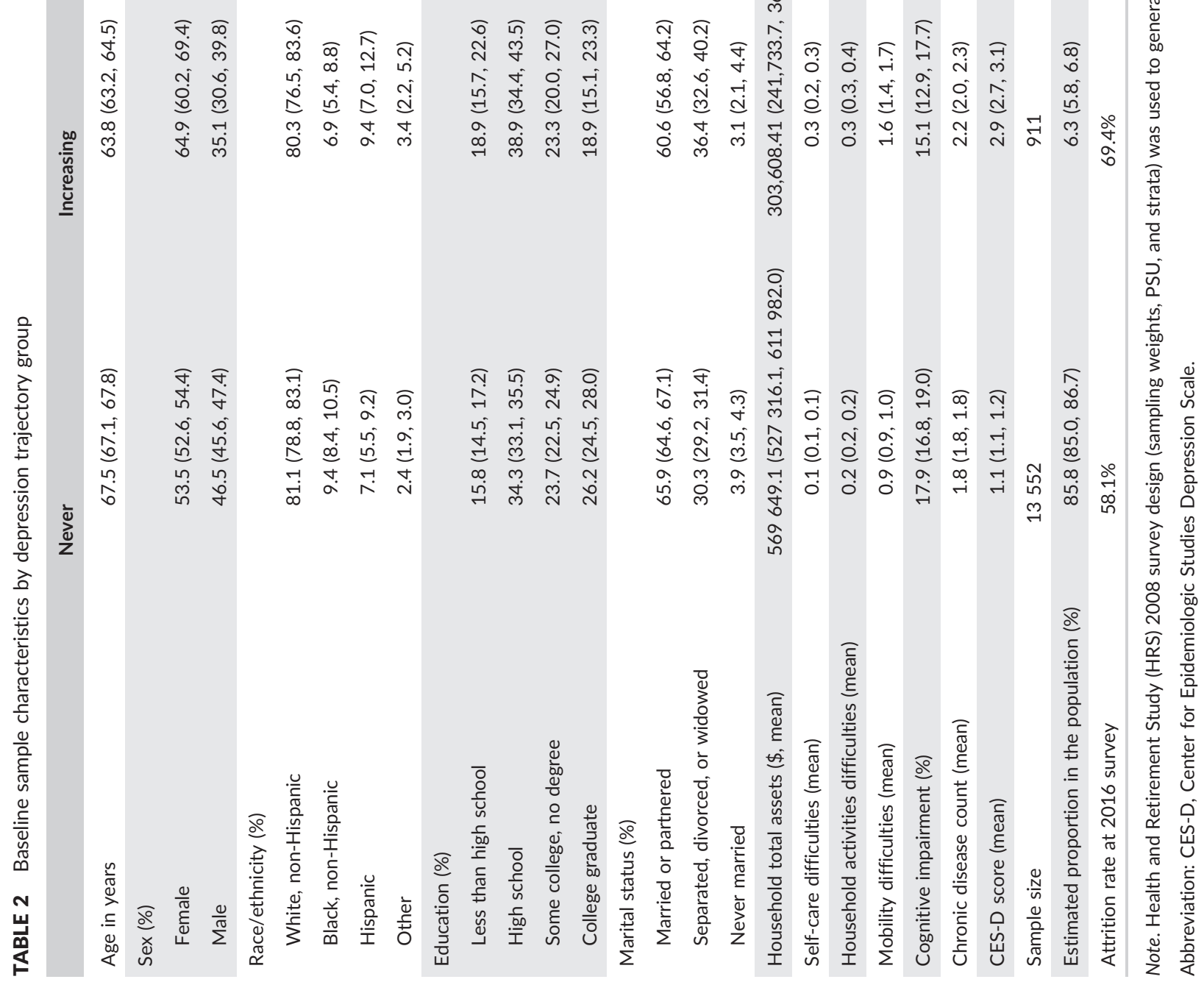


TABLE 3 Multinomial logistic regression predicting depression trajectory group as a function of baseline characteristics

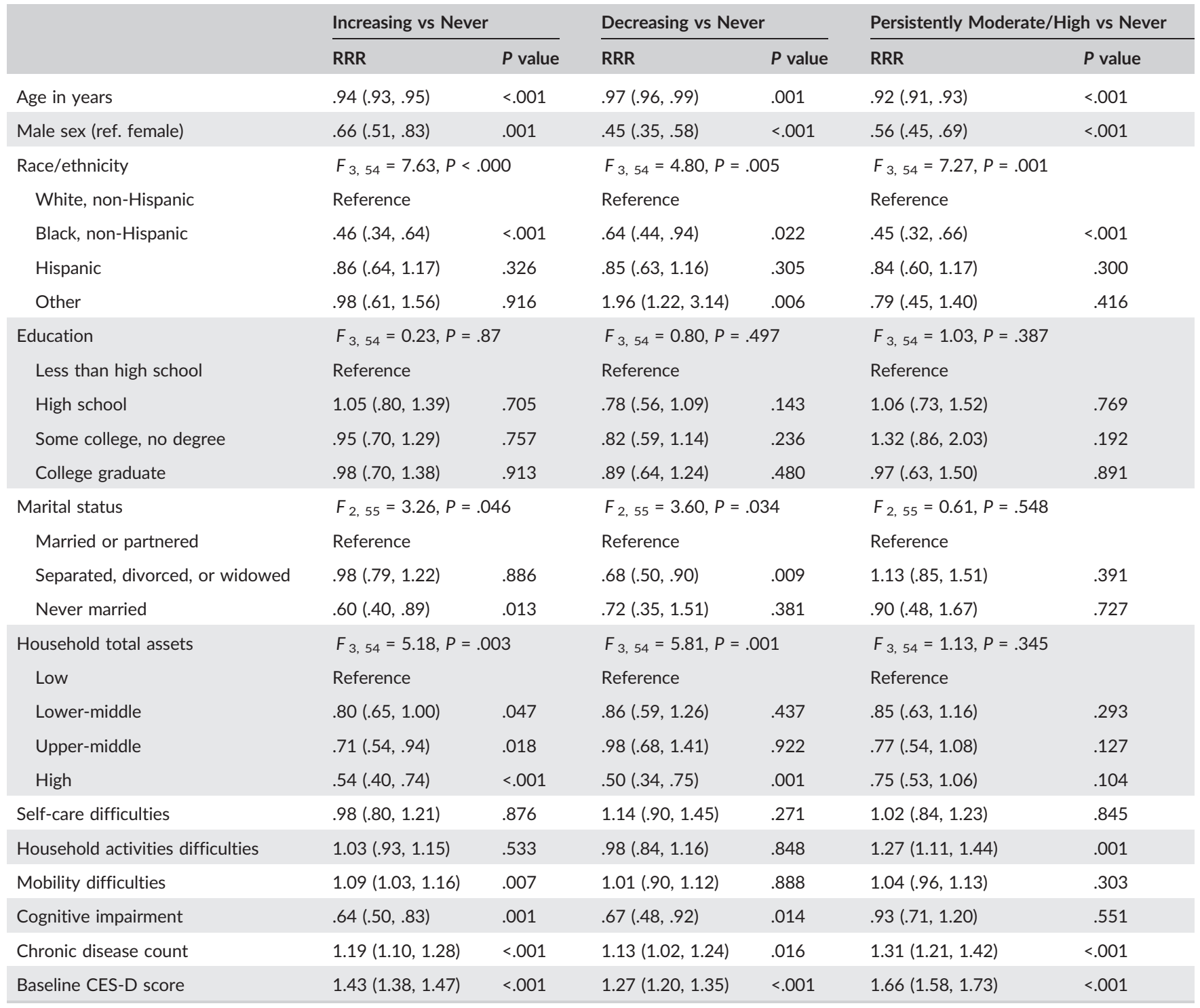

Abbreviations: CES-D, Center for Epidemiologic Studies Depression Scale; RRR, relative risk ratio.

living ( $A D L$ ) disability, which has been linked to unfavorable depression course in previous studies. ${ }^{8,16}$ A population-based study in the United States has shown that trait-like (ie, stable traits) outdoor mobility limitation was strongly associated with persistent depressive symptoms over time and that increased limitation in outdoor mobility predicted worsening depressive symptoms 1 year later. ${ }^{46}$ In another study that looked at a sample of noninstitutionalized older adults from Montpellier, France, having any mobility limitation that interfered with the ability to do heavy housework, walk half a mile, and climb stairs was associated with a four-to-five-fold increase in the odds of having increasing or chronic depressive symptoms. ${ }^{9}$ However, the French study did not include limitations in other self-care and household activities in its analysis and therefore could not delineate the independent impact of mobility limitations.

In bivariate analysis, the prevalence of cognitive impairment was the highest in the persistently moderate/high risk group, which is in line with the evidence that cognitive deficit is common in geriatric depression. In multivariable analysis, however, cognitive impairment was significantly associated with a lower risk of being on "increasing" and "decreasing" depression trajectories. This finding appears to contradict the convergent evidence that shows impairment in different cognitive domains, such as executive function, attention, memory, and psychomotor speed, during the acute phase of major depression. Studies have also reported that the impairment observed in the acute phase of depression may be long-lasting despite reduction in depressive symptoms. ${ }^{47}$ Cognitive dysfunction, particularly executive dysfunction, is common in geriatric depression and increases the risk for poor response to antidepressants. ${ }^{48}$ However, findings on the association between cognitive impairment and the clinical course of depression have been divergent in both clinical- and population-based samples, with some studies reporting significant associations ${ }^{19,49}$ and some reporting null findings. ${ }^{8,50}$ Separate processes may be involved in specific domains of cognition and response to depression treatment. ${ }^{51}$ The cognitive assessments in our study primarily measured 
short-term memory and mental tracking, which might explain our divergent findings regarding the role of cognitive functioning in depression course. Missing data may be another explanation for the unexpected finding regarding the role of cognitive impairment. CIDISF was not administered to proxies who responded to the HRS interviews when sample persons were unavailable, often due to an illness or impairment. Therefore, persons with cognitive impairment are more likely to have missing data on depression and have been excluded from our analysis.

Our results should be interpreted in consideration of several limitations. The CIDI-SF used in the HRS assesses 12-month major depression, not lifetime major depression. Therefore, our study provides limited information regarding the course of depression among older adults with a history of major depression. Our findings are best interpreted as patterns of changes in the occurrence of 12-month MDE over a relatively long period in middle-age and late-life. Moreover, HRS interviews are administered biennially whereas CIDI-SF probes symptoms in the past 12 months. Occurrences of MDE outside of the CIDI-SF reference periods may have occurred, leading to an overestimate of people on the "never" trajectory. Furthermore, persons with cognitive impairment are more likely to have missing data. In addition, predictors of depression trajectories were measured at baseline. The use of distant predictors may have contributed to the limited number of significant predictors from multivariable analysis. Finally, a small number of participants were assigned to the decreasing, increasing, and moderate/high groups, which might have made the regression analysis underpowered to detect moderate to small effect sizes.

\section{5 | CONCLUSION}

Considering our study findings, future studies should distinguish mobility limitations from other types of functional impairment and investigate the mechanisms whereby mobility limitation leads to unfavorable depression course. Studies using a more comprehensive cognitive assessment covering executive function, attention, language, and other domains will help clarify the association of cognitive functioning and long-term course of major depression in older adults. Our study included a limited set of predictors. Future research is needed to examine potential protective factors against unfavorable depression course, such as social support, social connectedness, productive engagement, and healthy lifestyle behaviors, to inform preventions and treatments. We did not assess treatment utilization by depression trajectories. Future studies should investigate the extent of treatment utilization and how this affects the depression course to identify treatment gaps and potential targets for service delivery innovation.

\section{ACKNOWLEDGEMENT}

This work was supported by a grant from the National Institutes of Health, University of Michigan Older Americans Independence Center Research Education Core (Grant number: AG024824).

\section{CONFLICT OF INTEREST}

None declared.

\section{DATA AVAILABILITY STATEMENT}

The data that support the findings of this study are openly available in the Health and Retirement Study web portal at http://hrsonline.isr. umich.edu/. These data were derived from the following public data products: HRS Biennial Data Products 2008 through 2016 HRS Core files and the RAND Contributed Files RAND HRS Longitudinal File 2014 (v.3).

\section{ORCID}

Xiaoling Xiang (D) https://orcid.org/0000-0002-4926-4707

\section{REFERENCES}

1. Beekman ATF, Copeland J, Prince MJ. Review of community prevalence of depression in later life. Br J Psychiatry. 1999;174(4):307-311. https://doi.org/10.1192/bjp.174.4.307

2. Seitz D, Purandare N, Conn D. Prevalence of psychiatric disorders among older adults in long-term care homes: a systematic review. Int Psychogeriatrics. 2010;22(7):1025-1039. https://doi.org/10.1017/ S1041610210000608

3. Mueller TI, Kohn R, Leventhal N, et al. The course of depression in elderly patients. Am J Geriatr Psychiatry. 2004;12(1):22-29. https:// doi.org/10.1097/00019442-200401000-00003

4. Beekman ATF, Deeg DJH, Braam AW, Smit JH, Van Tilburg W. Consequences of major and minor depression in later life: a study of disability, well-being and service utilization. Psychol Med. 1997;27(6):1397-1409. https://doi.org/10.1017/S003329179700 5734

5. Alexopoulos GS. The vascular depression hypothesis: 10 years later. Biol Psychiatry. 2006;60(12):1304-1305. https://doi.org/10.1016/j. biopsych.2006.09.006

6. Musliner KL, Munk-Olsen T, Eaton WW, Zandi PP. Heterogeneity in long-term trajectories of depressive symptoms: patterns, predictors and outcomes. J Affect Disord. 2016;192:199-211. https://doi.org/ 10.1016/j.jad.2015.12.030

7. Andreescu C, Chang CCH, Mulsant BH, Ganguli M. Twelve-year depressive symptom trajectories and their predictors in a community sample of older adults. Int Psychogeriatrics. 2008;20(2):221-236. https://doi.org/10.1017/S1041610207006667

8. Byers AL, Vittinghoff E, Lui LY, et al. Twenty-year depression trajectories among older women. Arch Gen Psychiatry. 2012;69(10):1073-1079. https://doi.org/10.1001/archgenpsychiatry. 2012.43

9. Carrière I, Farré A, Proust-Lima C, Ryan J, Ancelin ML, Ritchie K Chronic and remitting trajectories of depressive symptoms in the elderly. Characterisation and risk factors. Epidemiol Psychiatr Sci. 2017;26(2):146-156. https://doi.org/10.1017/S2045796015001122

10. Park MJ. Impact of social capital on depression trajectories of older women in Korea. Aging Ment Heal. 2017;26(2):146-156. https://doi. org/10.1080/13607863.2015.1088511

11. Kuchibhatla MN, Fillenbaum GG, Hybels CF, Blazer DG. Trajectory classes of depressive symptoms in a community sample of older adults. Acta Psychiatr Scand. 2012;125(6):492-501. https://doi.org/10.1111/ j.1600-0447.2011.01801.x 
12. Liang J, Xu X, Quiñones AR, Bennett JM, Ye W. Multiple trajectories of depressive symptoms in middle and late life: racial/ethnic variations. Psychol Aging. 2011;26(4):761-777. https://doi.org/10.1037/ a0023945

13. Saeed Mirza S, Ikram MA, Freak-Poli R, Hofman A, Rizopoulos D, Tiemeier H. 12 year trajectories of depressive symptoms in community-dwelling older adults and the subsequent risk of death over 13 years. Journals Gerontol - Ser a Biol Sci Med Sci. 2018;73(6):820-827. https://doi.org/10.1093/gerona/glx215

14. Montagnier D, Dartigues JF, Rouillon F, Pérés K, Falissard B, Onen F. Ageing and trajectories of depressive symptoms in communitydwelling men and women. Int J Geriatr Psychiatry. 2014;29 (7):720-729. https://doi.org/10.1002/gps.4054

15. Tampubolon G, Maharani A. When did old age stop being depressing? Depression trajectories of older Americans and Britons 2002-2012. Am J Geriatr Psychiatry. 2017;25(11):1187-1195. https://doi.org/ 10.1016/j.jagp.2017.06.006

16. Rote S, Chen NW, Markides K. Trajectories of depressive symptoms in elderly Mexican Americans. J am Geriatr Soc. 2015;63(7):1324-1330. https://doi.org/10.1111/jgs.13480

17. Melchior M, Chastang JF, Head J, et al. Socioeconomic position predicts long-term depression trajectory: a 13-year follow-up of the GAZEL cohort study. Mol Psychiatry. 2013;18(1):112-121. https://doi. org/10.1038/mp.2011.116

18. Cui XJ, Lyness JM, Tang W, Tu X, Conwell Y. Outcomes and predictors of late-life depression trajectories in older primary care patients. Am J Geriatr Psychiatry. 2008;16(5):406-415. https://doi.org/10.1097/01. JGP.0000308881.22956.27

19. Holmes SE, Esterlis I, Mazure CM, et al. Trajectories of depressive and anxiety symptoms in older adults: a 6-year prospective cohort study. Int J Geriatr Psychiatry. 2018;33(2):405-413. https://doi.org/10.1002/ gps.4761

20. Lyness JM, Caine ED, King DA, Conwell Y, Duberstein PR, Cox C. Depressive disorders and symptoms in older primary care patients: one-year outcomes. Am J Geriatr Psychiatry. 2002;10(3):275-282. https://doi.org/10.1097/00019442-200205000-00006

21. Eaton WW, Shao H, Nestadt G, Lee BH, Bienvenu OJ, Zandi P. Population-based study of first onset and chronicity in major depressive disorder. Arch Gen Psychiatry. 2008;65(5):513-520. https://doi.org/ 10.1001/archpsyc.65.5.513

22. Rhebergen D, Lamers F, Spijker J, De Graaf R, Beekman ATF, Penninx BWJH. Course trajectories of unipolar depressive disorders identified by latent class growth analysis. Psychol Med. 2012;42(7):1383-1396. https://doi.org/10.1017/S0033291711002509

23. Haigh EAP, Bogucki OE, Sigmon ST, Blazer DG. Depression among older adults: a 20-year update on five common myths and misconceptions. Am J Geriatr Psychiatry. 2018;26(1):107-122. https://doi.org/ 10.1016/j.jagp.2017.06.011

24. Mojtabai R, Olfson M. Major depression in community-dwelling middle-aged and older adults: prevalence and 2- and 4-year followup symptoms. Psychol Med. 2004;34(4):623-634. https://doi.org/ $10.1017 /$ S0033291703001764

25. Hybels CF, Pieper CF, Blazer DG, Steffens DC. Heterogeneity in the three-year course of major depression among older adults. Int J Geriatr Psychiatry. 2016;31(7):775-782. https://doi.org/10.1002/gps.4391

26. Ojagbemi A, Abiona T, Luo Z, Gureje O. Symptomatic and functional recovery from major depressive disorder in the Ibadan study of ageing. Am J Geriatr Psychiatry. 2018;26(6):657-666. https://doi.org/10.1016/ j.jagp.2017.12.011

27. Health and Retirement Study, HRS Biennial Data Products and RAND Contributed Files public use dataset. Produced and distributed by the
University of Michigan with funding from the National Institute on Aging (grant number NIA U01AG009740). Ann Arbor, MI, 2019.

28. Kessler RC, Andrews G, Mroczek D, Ustun B, Wittchen H-U. The World Health Organization composite international diagnostic interview short-form (CIDI-SF). Int J Methods Psychiatr Res. 1998;7(4):171-185. https://doi.org/10.1002/mpr.47

29. Steffick DE. Documentation of affective functioning measures in the Health and Retirement Study. Ann Arbor, MI: University of Michigan; 2000.

30. Brandt J, Spencer M, Folstein MF. The telephone interview for cognitive status. Neuropsychiatry Neuropsychol Behav Neurol. 1988;1(2): 1111-117.

31. Crimmins EM, Kim JK, Langa KM, Weir DR. Assessment of cognition using surveys and neuropsychological assessment: the health and retirement study and the aging, demographics, and memory study. Journals Gerontol Ser B Psychol Sci Soc Sci. 2011;66B(Supplement 1): i162-i171. https://doi.org/10.1093/geronb/gbr048

32. Kohout FJ, Berkman LF, Evans DA, Cornoni-Huntley J. Two shorter forms of the CES-D depression symptoms index. J Aging Health. 1993;5(2):179-193. https://doi.org/10.1177/089826439300500202

33. Haviland AM, Jones BL, Nagin DS. Group-based trajectory modeling extended to account for nonrandom participant attrition. Sociol Methods Res. 2011;40(2):367-390. https://doi.org/10.1177/004912 4111400041

34. Nagin D. Group-Based Modeling of Development. Cambridge, MA: Harvard University Press; 2005.

35. Jones BL, Nagin DS. Advances in group-based trajectory modeling and an SAS procedure for estimating them. Sociol Methods Res. 2007;35(4):542-571. https://doi.org/10.1177/0049124106292364

36. Bruin MC, Comijs HC, Kok RM, Van der Mast RC, Van den Berg JF. Lifestyle factors and the course of depression in older adults: a NESDO study. Int J Geriatr Psychiatry. 2018;33(7):1000-1008. https://doi.org/ 10.1002/gps.4889

37. Van Orden KA, Chen S, O'Riley A, Conwell Y. Course of late-life depression in China is chronic and unremitting. Int J Geriatr Psychiatry. 2015;30(4):409-415. https://doi.org/10.1002/gps.4151

38. Steinert C, Hofmann M, Kruse J, Leichsenring F. The prospective longterm course of adult depression in general practice and the community. A systematic literature review. J Affect Disord. 2014;152:65-75. https://doi.org/10.1016/j.jad.2013.10.017

39. Spijker J, De Graaf R, Bijl RV, Beekman ATF, Ormel J, Nolen WA. Determinants of persistence of major depressive episodes in the general population. Results from the Netherlands mental health survey and incidence study (NEMESIS). J Affect Disord. 2004;81(3):231-240. https://doi.org/10.1016/j.jad.2003.08.005

40. Dunlop DD, Song J, Lyons JS, Manheim LM, Chang RW. Racial/ethnic differences in rates of depression among preretirement adults. Am J Public Health. 2003 Nov;93(11):1945-1952.

41. Riolo SA, Nguyen TA, Greden JF, King CA. Prevalence of depression by race/ethnicity: findings from the National Health and Nutrition Examination Survey III. Am J Public Health. 2005 Jun;95(6):998-1000.

42. Cohen $\mathrm{Cl}$, Goh KH, Gustave M. A prospective study of outcome and predictors of subclinical and clinical depression in an older biracial sample of psychiatric outpatients. J Affect Disord. 2010;121(3): 204-211. https://doi.org/10.1016/j.jad.2009.05.021

43. Ryff CD. Happiness is everything, or is it? Explorations on the meaning of psychological well-being. J Pers Soc Psychol. 1989;57(6):1069-1081. https://doi.org/10.1037/0022-3514.57.6.1069

44. Te LC, Yeh CJ, Lee MC, et al. Leisure activity, mobility limitation and stress as modifiable risk factors for depressive symptoms in the elderly: 
results of a national longitudinal study. Arch Gerontol Geriatr. 2012; 54(2):e221-e229. https://doi.org/10.1016/j.archger.2011.06.014

45. Polku H, Mikkola TM, Portegijs E, et al. Life-space mobility and dimensions of depressive symptoms among community-dwelling older adults. Aging Ment Heal. 2015;19(9):781-789. https://doi.org/10. 1080/13607863.2014.977768

46. Xiang X, An R, Oh H. The bidirectional relationship between depressive symptoms and homebound status among older adults. Journals Gerontol Ser B. 2018. https://doi.org/10.1093/geronb/gbx180

47. Hammar Å, Årdal G. Cognitive functioning in major depression-a summary. Front Hum Neurosci. 2009;3. https://doi.org/10.3389/neuro. 09.026.2009

48. Alexopoulos GS, Kiosses DN, Heo M, Murphy CF, Shanmugham B, Gunning-Dixon F. Executive dysfunction and the course of geriatric depression. Biol Psychiatry. 2005;58(3):204-210. https://doi.org/10. 1016/j.biopsych.2005.04.024

49. Mojtabai R, Olfson M. Cognitive deficits and the course of major depression in a cohort of middle-aged and older community-dwelling adults. J am Geriatr Soc. 2004;52(7):1060-1069. https://doi.org/ 10.1111/j.1532-5415.2004.52302.x

50. Bogner HR, Morales KH, Reynolds CF, Cary MS, Bruce ML. Prognostic factors, course, and outcome of depression among older primary care patients: the PROSPECT study. Aging Ment Heal. 2012;16(4):452-461. https://doi.org/10.1080/13607863.2011. 638904

51. Bogner HR, Bruce ML, Reynolds CF III, et al. The effects of memory, attention, and executive dysfunction on outcomes of depression in a primary care intervention trial: the PROSPECT study. Int J Geriatr Psychiatry. 2007;22(9):922-929. https://doi.org/10.1002/gps.1767

How to cite this article: Xiang $X$, Cheng J. Trajectories of major depression in middle-aged and older adults: A population-based study. Int J Geriatr Psychiatry. 2019;34: 1506-1514. https://doi.org/10.1002/gps.5161 